\title{
Is clinical target volume necessary? - a failure pattern analysis in patients with locally advanced non-small cell lung cancer treated with concurrent chemoradiotherapy using intensity-modulated radiotherapy technique
}

\author{
Liqing Zou ${ }^{1,2 \#}, \mathrm{Li} \mathrm{Chu}^{1,2 \#}$, Fan $\mathrm{Xia}^{1,2}$, Lijun Zhou ${ }^{1,2}$, Xi Yang ${ }^{1,2}$, Jianjiao $\mathrm{Ni}^{1,2}$, Junchao Chen ${ }^{1,2}$, \\ Zhengfei Zhu ${ }^{1,2,3}$
}

${ }^{1}$ Department of Radiation Oncology, Fudan University Shanghai Cancer Center, Shanghai, China; ${ }^{2}$ Department of Oncology, Fudan University Shanghai Medical College, Shanghai, China; ${ }^{3}$ Institute of Thoracic Oncology, Fudan University, Shanghai, China

Contributions: (I) Conception and design: Z Zhu; (II) Administrative support: Z Zhu; (III) Provision of study materials or patients: Z Zhu; (IV) Collection and assembly of data: L Zou, L Chu; (V) Data analysis and interpretation: L Zou, L Chu; (VI) Manuscript writing: All authors; (VII) Final approval of manuscript: All authors.

"These authors contributed equally to this work.

Correspondence to: Zhengfei Zhu. Department of Radiation Oncology, Fudan University Shanghai Cancer Center, Shanghai 200032, China; Department of Oncology, Shanghai Medical College, Fudan University, Shanghai 200032, China; Institute of Thoracic Oncology, Fudan University, Shanghai 200032, China. Email: fuscczzf@163.com.

Background: Our previous dosimetric study showed that for locally advanced non-small cell lung cancer (LA-NSCLC), radiotherapy with intensity-modulated radiotherapy (IMRT) technique could deliver sufficient dose coverage to subclinical regions and reduce the dose to normal tissues with the omission of clinical target volume (CTV). To further clinically validate this strategy, we conducted the current study to analyze the failure pattern for patients with LA-NSCLC treated with concurrent chemotherapy and CTVomitted IMRT. We also investigated the effects of target volumes on lymphopenia during radiotherapy to further test the potential benefits of CTV omission in anti-tumor immunotherapy.

Methods: A total of 63 patients with LA-NSCLC treated with CTV-omitted IMRT with concurrent chemotherapy were enrolled in this study. Their planning target volume (PTV) (also PTV-g) was expanded directly from gross tumor volume (GTV). A virtual CTV was expanded from GTV, and the PTV generated from virtual CTV was named planning target volume with CTV expansion (PTV-c). Treatment failures were divided into local, regional, and distant failures, and local-regional recurrences were classified into inside PTV-g (IN-PTV-g), between PTV-g and PTV-c (PTV-g-c), and outside PTV-c (OUT-PTV-c). The relationship between lymphopenia during radiotherapy and the target volumes was also evaluated using Spearman's correlation analysis.

Results: Among the 60 patients with detailed follow-up data for recurrences, 46 (76.7\%) experienced recurrences, with 18 (30.0\%) being local recurrence, 5 (8.4\%) being regional failure, and $33(55.0 \%)$ being distant failure. For the 21 patients with local-regional recurrences, 16, 6, and 1 were IN-PTV-g, OUTPTV-c, and PTV-g-c recurrences, respectively. Lymphopenia during radiotherapy was associated with both GTV and PTV, with larger volumes linked to severe lymphopenia.

Conclusions: CTV omission is feasible for LA-NSCLC treated with concurrent chemoradiotherapy and does not compromise failure inside the subclinical region. The radiation volumes were associated with lymphopenia during radiotherapy, with larger volumes related to severe lymphopenia. This finding supports the further exploration of CTV omission for immunotherapy.

Keywords: Non-small cell lung cancer (NSCLC); stage III; clinical target volume (CTV); intensity-modulated radiotherapy (IMRT); recurrence 
Submitted Feb 25, 2020. Accepted for publication Jul 27, 2020.

doi: $10.21037 /$ tlcr-20-523

View this article at: http://dx.doi.org/10.21037/tlcr-20-523

\section{Introduction}

For $20-30 \%$ of patients with locally advanced non-small cell lung cancer (LA-NSCLC) (AJCC TNM-8, stage III), tumors are unresectable and definitive concurrent chemoradiation is the standard care for unresectable LANSCLC $(1,2)$. Radiotherapy is an important therapeutic strategy with radical cure potential for patients with LANSCLC, and intensity-modulated radiotherapy (IMRT) has been mainly recommended because it can achieve satisfying dose coverage of tumor while sparing normal tissue (3-7). For IMRT, radiation target contouring is crucial. The planning target volume (PTV) is generated by procedurally expanding gross tumor volume (GTV), clinical target volume (CTV), and internal target volume, and the radical radiation dose is then prescribed to PTV in the definitive radiotherapy for LA-NSCLC. However, CTV is the tissue volume that contains GTV and subclinical microscopic malignant lesions, and the required doses for subclinical disease eradication are lower than those used to control gross tumors in patients with common epithelial tumors $(8,9)$. Given this finding, we hypothesized that the dosage prescribed to subclinical lesions in traditional target-contouring and a dose-prescription manner is higher than necessary. This hypothesis prompted us to explore the feasibility of omitting CTV in patients with LA-NSCLC. Our previous dosimetric study (10) showed that radiotherapy with IMRT technique can deliver sufficient dose coverage to subclinical diseases and reduce the dose to normal tissues even with CTV omission. To further validate the strategy in clinical practice, we conducted the current study to analyze the failure pattern for patients with LANSCLC treated with concurrent chemotherapy and CTVomitted IMRT. We focused on the rate of recurrences in subclinical areas to directly test the feasibility of CTV omission.

At present, anti-tumor immunotherapy has become a necessity for LA-NSCLC treatment. Based on the results of the PACIFIC study $(11,12)$ and the outcomes of consolidation treatment with the PD-L1 antibody, durvalumab after concurrent chemoradiotherapy has become the standard of care for patients with unresectable LA-NSCLC.

Immune system integrity is vital for stimulating effective anti-tumor immune effects during immunotherapy (13). However, lymphocytes, as part of systematic immune cells, are extremely sensitive to radiation (14) due to the direct irradiation of lymph nodes and to circulating lymphocytes (CLs) traversing through the radiation field (15). Radiationinduced lymphopenia (RIL) occurs in $40 \%$ to $70 \%$ of patients undergoing radiotherapy (16). The nadir of absolute lymphocyte counts (ALC) during radiotherapy is associated with poor survival in patients with NSCLC according to multiple studies (17-19), and a low ALC nadir during radiotherapy can also indicate a reduced response to immunotherapy for NSCLC $(20,21)$. Thus, in addition to the routine toxicities caused by radiotherapy, RIL in immunotherapy must be further investigated. Accordingly, the present study evaluated the effects of target volumes on RIL during radiotherapy through several parameters reflecting lymphocyte counts with the aim of further testing the potential benefits of CTV omission in anti-tumor immunotherapy.

We present the following article in accordance with the STROBE reporting checklist (available at http://dx.doi. org/10.21037/tlcr-20-523).

\section{Methods}

\section{Patients and treatment}

All patients with NSCLC treated with definitive chemoradiotherapy in our institution between 2013 and 2018 were reviewed, and patients were selected in accordance with the following criteria: pathologically confirmed stage III NSCLC according to the American Joint Committee on Cancer (8th edition); treated with concurrent chemoradiotherapy (at least 2 cycles of platinum-based chemotherapy delivered during radiotherapy, at least 4 cycles if weekly chemotherapy protocols were delivered); radiotherapy performed with IMRT technique at 60 Gy total dose in 30 fractionations at 2 Gy per fraction once per day for 5 fractions per week; and PTV generated directly from GTV without CTV expanding (PTV-g hereinafter), as previously described (10). Exclusion criteria was as follows: patients with other tumor at diagnosis of NSCLC; patients receiving neoadjuvant, adjuvant, and palliative radiotherapy; patients not treated with concurrent chemoradiotherapy; radiotherapy prescription was not IMRT with the dose of 60 Gy in 30 


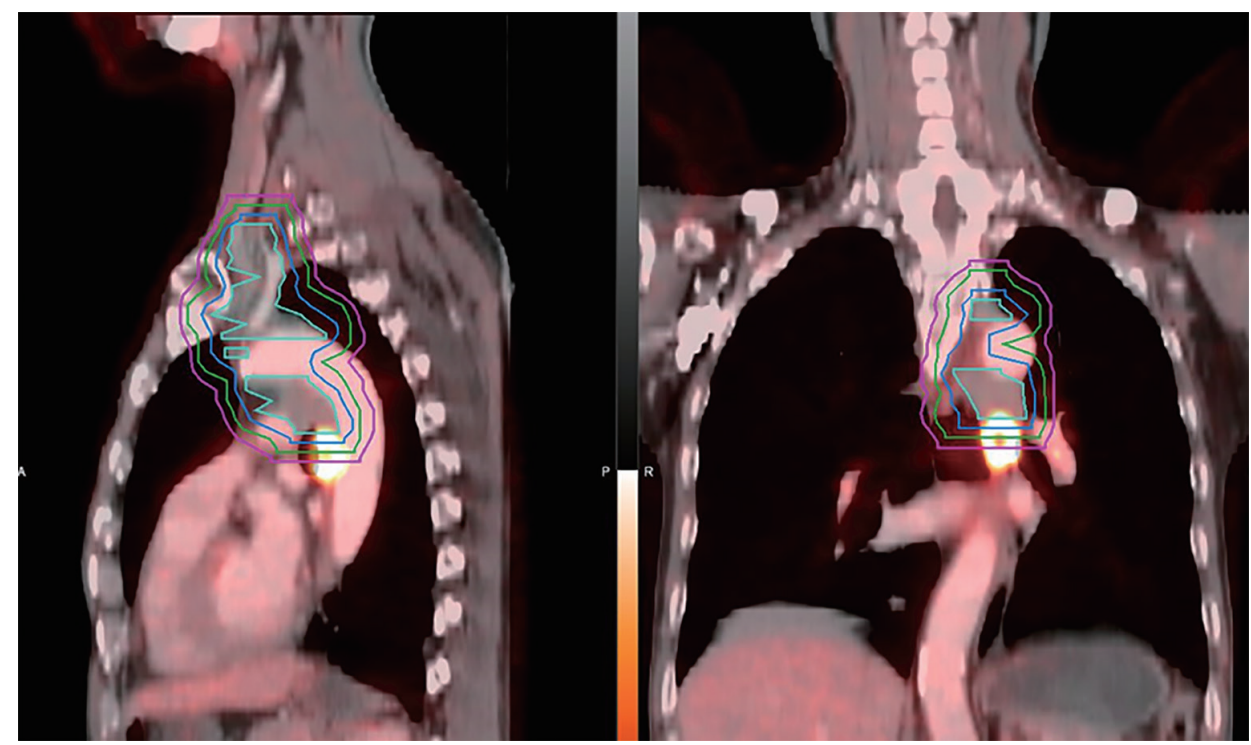

Figure 1 Failure location is based on the original treatment planning from coronal and sagittal CT) scans, with matched post-treatment PET scans demonstrating recurrence. The locations are visible as bright spots on the fusion PET/CT scans. The lines of different colors from inside to outside represent GTV, CTV, PTV-g, and PTV-c, respectively. The pictures are from a case of PTV-g-c recurrence in which the center of the recurrence lesion is located between PTV-g and PTV-c. CT, computed tomography; PET, positron emission tomography; GTV, gross tumor volume; CTV, clinical target volume; PTV-g, planning target volume without CTV expansion; PTV-c, planning target volume with CTV expansion.

fractions; and patients who didn't complete the prescribed dose of chemoradiotherapy were also excluded. The study was conducted in accordance with the Declaration of Helsinki (as revised in 2013). The study was approved by institutional ethics board of Fudan University Shanghai Cancer Center (No. IRB\#090978-2). Patient consent was waived because this study was a retrospective study.

\section{Radiotherapy and follow-up}

Patients' immobilization, radiation target contour and generation, dose requirements for treatment planning, and radiation techniques are described elsewhere (10). In general, the patients were followed up every 3 months for 2 years, every 6 months for 2-5 years, and once per year thereafter. During follow-up, the medical history, physical examination, chest computed tomography (CT) scans, and abdominal ultrasound or CT of the patients were assessed. Other tests, such as brain magnetic resonance imaging, bone scanning, and positron emission tomography (PET) scans were performed at the discretion of the treating physicians. Follow-up through telephone interviews was also used as a supplementary measure.

\section{Patterns of failure analysis}

In this analysis, we focused on the patterns of first failure, which were first divided into local, regional, and distant failures. Local failure included the primary tumor and the originally metastatic regional lymph nodes (LNs); regional failure included the regional LN areas, including hilar, mediastinal, and supraclavicular LNs, but excluded the originally metastatic regional LNs; and everywhere else was considered distant failure. We then classified the local and regional (local-regional) failures into inside PTV-g (INPTV-g), between PTV-g and PTV-c (PTV-g-c), and outside PTV-c (OUT-PTV-c). PTV-c was generated as previously described (10): we first created a virtual CTV by expanding 6 and $8 \mathrm{~mm}$ from GTV for squamous cell carcinoma and adenocarcinoma, respectively; 3 and $5 \mathrm{~mm}$ for the nodes with a $<2$ and $\geq 2 \mathrm{~cm}$ short axis, respectively, with the vertebral bodies, trachea, proximal bronchial trees, heart, large vessels, and esophagus being manually modified for exclusion; and PTV-c was then created from virtual CTV by considering the set-up error factors and organ motion (the margin was the same as that from GTV to PTV-g expansion for each patient). A schematic of the target volumes is shown in Figure 1. 
Local-regional failures were assessed on the basis of serial post-treatment chest CT. PET-CT and/or directed biopsy were performed for suspicious cases. Failure was scored from the first radiographic appearance of abnormality. Local-regional failure locations were further identified by fusing the current CT or PET-CT scans with the treatment plan CT scan (Figure 1).

\section{Correlation between RIL and target volumes}

Patients with medical records for weekly complete blood counts $(\mathrm{CBC})$ during radiotherapy were retrospectively reviewed. All CBC has followed the CBC SOP (standard operating procedure) of the laboratory in our center. The nadirs of absolute lymphocyte count (ALC) during definitive radiotherapy were recorded, along with the nadir of white blood cell (WBC), neutrophil (NE), and monocyte (MO) counts. Three relative lymphocyte indexes during radiotherapy were also investigated: percentage of lymphocytes (LY\%) nadir, ALC/NE baseline, and ALC/ALC baseline (within 3 weeks before the start of radiotherapy, $\mathrm{ALC}_{\mathrm{RT}} / \mathrm{ALC}_{\text {Baseline }}$ ). GTV and PTV-g volumes were acquired from the Pinnacle treatment planning system version 8.0 (Philips Medical Systems, Fitchburg, WI, USA) to test the relationship between target volumes and these parameters.

\section{Statistical analysis}

Statistical analysis was performed with Statistical Package for the Social Sciences (SPSS) version 19.0 (SPSS, Inc., Chicago, IL, USA). Continuous variables were summarized by descriptive statistics, including means, standard deviations, medians, and ranges. Categorical variables were tabulated by frequency and percentage. Overall survival (OS) and recurrence-free survival (RFS) rates were analyzed by KaplanMeier analysis. OS was defined as the time from the start of radiotherapy to death due to any cause. RFS was documented as the time from the start of radiotherapy to first recurrence or death. Patients alive at the time of last follow-up were allocated that date. Spearman's correlation coefficients (r) were used to assess univariate associations between laboratory minimums with the $\log 10$ of GTV and the $\log 10$ of PTV-g being used to convert GTV and PTV-g into a normal distribution.

\section{Results}

\section{Patient demographics and $O S$}

A total of 63 patients were enrolled in our study. The characteristics of 63 patients are shown in Table 1. One patient was lost to follow-up for OS. For the remaining 62 patients, the median follow-up time was 20.2 months (range, 3.668.3 months), the median OS was 33.0 months [ $95 \%$ confidence interval (CI): 25.3-40.7 months], and the 1-, 2-, and 3-year survival rates were $82.3 \%, 60.4 \%$, and $46.3 \%$, respectively. The Kaplan-Meier estimate of OS is shown in Figure $2 A$.

\section{Patterns of first failure}

A total of 3 patients were lost to follow-up for recurrence status. The median RFS for the remaining 60 patients was 9.0 months (95\% CI: 6.4-11.6 months), and the 1-, 2 -, and 3-year RFS rates were $41.1 \%, 25.6 \%$, and $25.6 \%$, respectively. The Kaplan-Meier curve of RFS is shown in Figure 2B. Among these patients, 46 (76.7\%) patients experienced recurrences, with 18 (30.0\%), 5 (8.4\%), and 33 (55.0\%) experiencing local recurrence, regional failure, and distant failure, respectively. The numbers for each type of failures are shown in Figure $3 \mathrm{~A}$. The distribution of the localregional failure is illustrated in Figure 3B. For the 21 patients who experienced local-regional recurrences at the first failure, 16, 6, and 1 patient encountered IN-PTV-g, OUT-PTV-c, and PTV-g-c failures, respectively. Representative scans illustrating the case with PTV-g-c failure are shown in Figure 1.

\section{Association between lymphopenia during radiotherapy and radiation target volumes}

A total of 56 patients with accessible continuous weekly blood test results during radiotherapy were included. The median volumes of GTV and PTV-g were $118.32 \mathrm{~cm}^{3}$ (range, 14.48-393.96 $\mathrm{cm}^{3}$ ) and $424.12 \mathrm{~cm}^{3}$ (range, 74.18$1,033.58 \mathrm{~cm}^{3}$ ), respectively. A significant inverse correlation was observed between ALC nadir during radiotherapy and $\log 10(\mathrm{GTV})(\mathrm{r}=-0.346, \mathrm{P}=0.015)$ and $\log 10(\mathrm{PTV}-\mathrm{g})$ $(\mathrm{r}=-0.487, \mathrm{P}<0.001)$ in these patients (Figure 4). However, no significant associations were found between GTV and PTV-g with the nadir of total WBC, NE, or MO counts during radiotherapy (all $\mathrm{P}>0.05$, Figure S1). This finding suggests that lymphocytes were more sensitive to chemoradiotherapy than other peripheral blood cells, a result that is consistent with that of previous studies $(8,14)$. For the other parameters that reflect lymphocyte counts during radiotherapy, the LY\% nadir, ALC/NE nadir, and ALCRT/ALCB were all significantly correlated with log 10 (PTV-g), whereas LY\% nadir and ALC/NE nadir were 
Table 1 Patient baseline demographic data and treatment status

\begin{tabular}{|c|c|}
\hline Patient characteristics & $\mathrm{N}=63, \mathrm{~N}(\%)$ \\
\hline \multicolumn{2}{|l|}{ Age (years) } \\
\hline Median & 62 \\
\hline Range & $31-78$ \\
\hline$>70$ & $10(15.9)$ \\
\hline \multicolumn{2}{|l|}{ Gender } \\
\hline Male & $50(79.4)$ \\
\hline Female & $13(20.6)$ \\
\hline \multicolumn{2}{|l|}{ Clinical stage } \\
\hline IIIA & $25(39.7)$ \\
\hline IIIB & $30(47.6)$ \\
\hline IIIC & $8(12.7)$ \\
\hline \multicolumn{2}{|l|}{ T stage } \\
\hline 1 & $10(15.9)$ \\
\hline 2 & $21(33.3)$ \\
\hline 3 & $19(30.2)$ \\
\hline 4 & $13(20.6)$ \\
\hline \multicolumn{2}{|l|}{$\mathrm{N}$ stage } \\
\hline 1 & $5(7.9)$ \\
\hline 2 & $39(61.9)$ \\
\hline 3 & $20(31.7)$ \\
\hline \multicolumn{2}{|l|}{ Histological subtype } \\
\hline Squamous cell & $31(49.2)$ \\
\hline Adenocarcinoma & $21(33.3)$ \\
\hline NSCLC NOS & $11(17.5)$ \\
\hline \multicolumn{2}{|l|}{ Smoking status } \\
\hline Non-smokers & $22(34.9)$ \\
\hline Ever/current smokers & $41(65.1)$ \\
\hline \multicolumn{2}{|l|}{ Chemotherapy protocol } \\
\hline Docetaxel + platinum & $30(47.6)$ \\
\hline Pemetrexed + platinum & $21(33.3)$ \\
\hline Paclitaxel + platinum & $12(19.0)$ \\
\hline
\end{tabular}

NSCLC, non-small cell lung cancer; NOS, not otherwise specified. Data are presented as number of patients $(\mathrm{N})$, with percentages in parentheses. correlated with $\log 10$ (GTV) (Figure 5).

\section{Discussion}

In the current analysis, we found that only 1 out of 60 (1.7\%) patients experienced subclinical regional (PTV$\mathrm{g}$-c) recurrence after concurrent chemoradiotherapy at 60 Gy radical radiation dose for LA-NSCLC, even without the intended CTV implementation. This finding clinically supports our previous dosimetric study (10), which demonstrated the feasibility of omitting CTV in this scenario. We also found that RIL was associated with the target volumes, especially PTV-g, with larger volumes linked to severe RIL, indicating that the target volume reduction through CTV omission could maintain the integrity of the immune system that may potentially favor immunotherapy.

CTV omission did not compromise the control inside the subclinical region for several reasons. First, from the radiobiological perspective, the doses necessary to control the subclinical lesions of common epithelial tumors are lower than those used to eradicate gross tumors $(8,9)$. Thus, the low peripheral doses around gross tumors may sufficiently control the subclinical foci, which has been proven by our dosimetric study (10). The second reason can be deduced from the present status of treatment failure patterns for LA-NSCLC treated with concurrent chemoradiotherapy. Consistent with many previous studies (22-24), we found that major failure occurred in the distant areas, which may shadow over the local-regional recurrences; most local-regional recurrences occurred with IN-PTV-g rather than subclinical regions, implying that the current standard radiation dose for LA-NSCLC (60 Gy per 30 fractions) may be insufficient in eliminating gross tumors. Thus, in such a state, using radical radiation doses to treat the invisible subclinical lesions even when the visible gross tumors cannot be well controlled is excessive. Third, the concurrently used chemotherapy may be more effective for the subclinical lesions than the gross tumors. This speculation is based on the evidence that shows adjuvant chemotherapy has long-term survival benefit in patients with NSCLC (25-28).

Combining radiotherapy and immunotherapy can revolutionize cancer treatment. The success of the 

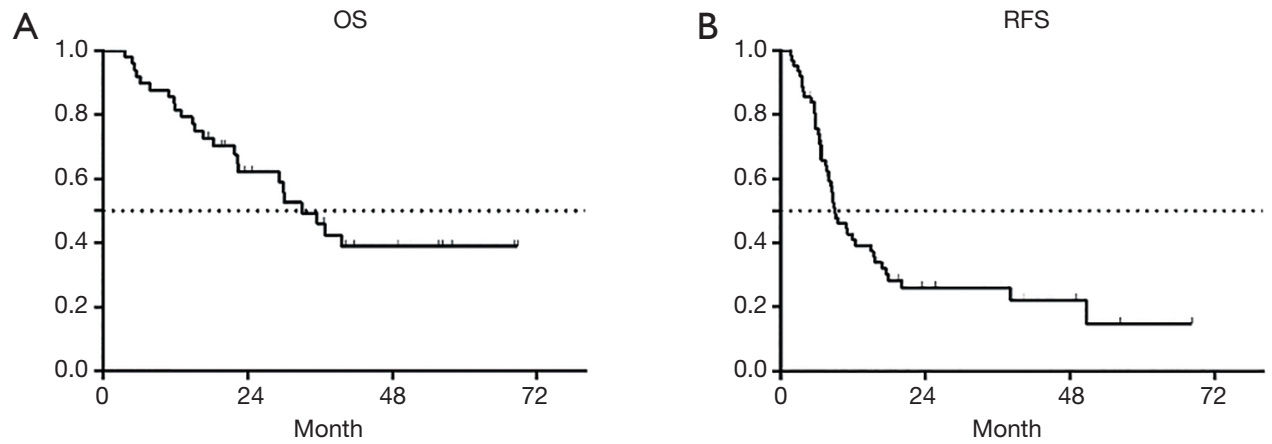

Figure 2 Kaplan-Meier curves for OS and RFS. (A) Kaplan-Meier plots for OS. (B) Kaplan-Meier plots for RFS. OS, overall survival; RFS, recurrence-free survival.
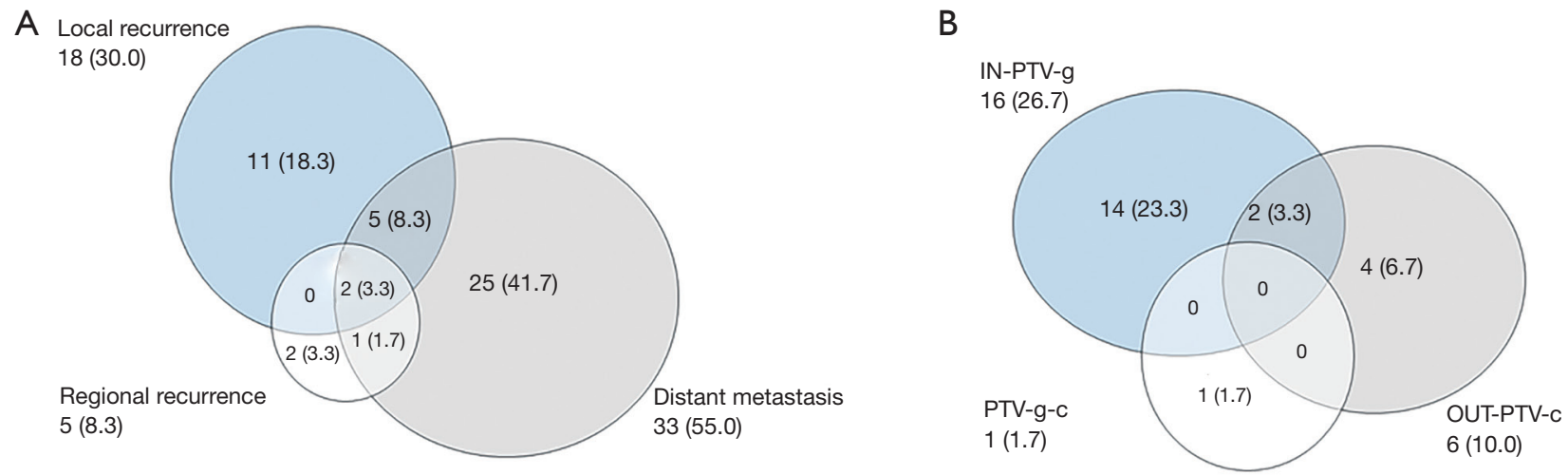

Figure 3 General and local-regional patterns of first failure are shown in A and B, respectively. Data are presented as the number of patients, with the percentages (shown in parentheses) relative to a total number of 60 patients (who had accessible follow-up data of local-regional recurrence).

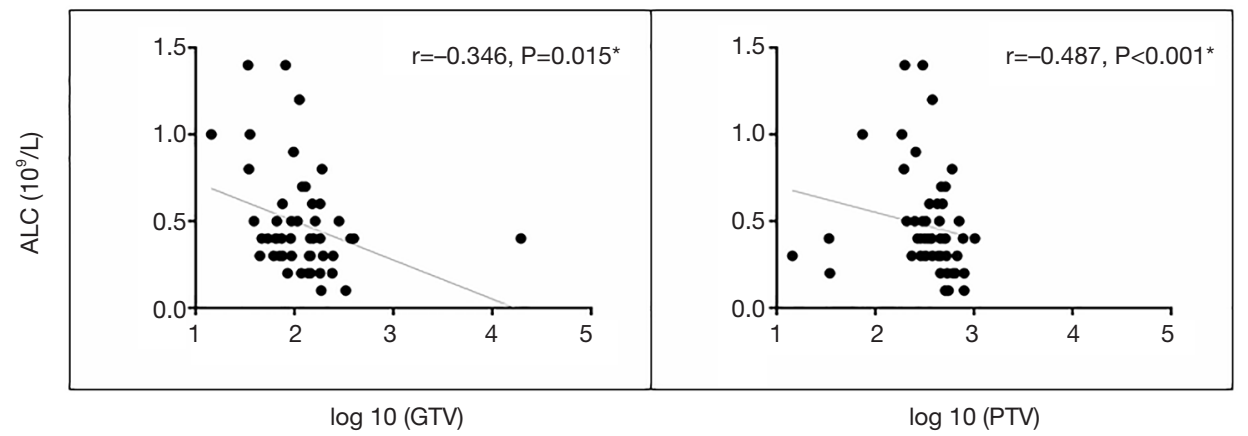

Figure 4 Correlations among ALC nadirs during radiotherapy and log 10 [GTV (cc)] and log 10 [PTV-g (cc)]. Spearman's correlation coefficients and corresponding $\mathrm{P}$ values are shown. A significant inverse correlation was observed between the ALC nadir during radiotherapy and $\log 10$ (GTV) (r=-0.346, P=0.015), and $\log 10$ (PTV-g) ( $\mathrm{r}=-0.487, \mathrm{P}<0.001)$. *, significantly correlated. ALC, absolute lymphocyte count; GTV, gross tumor volume; PTV-g, planning target volume without CTV expansion; r, correlation coefficient. 


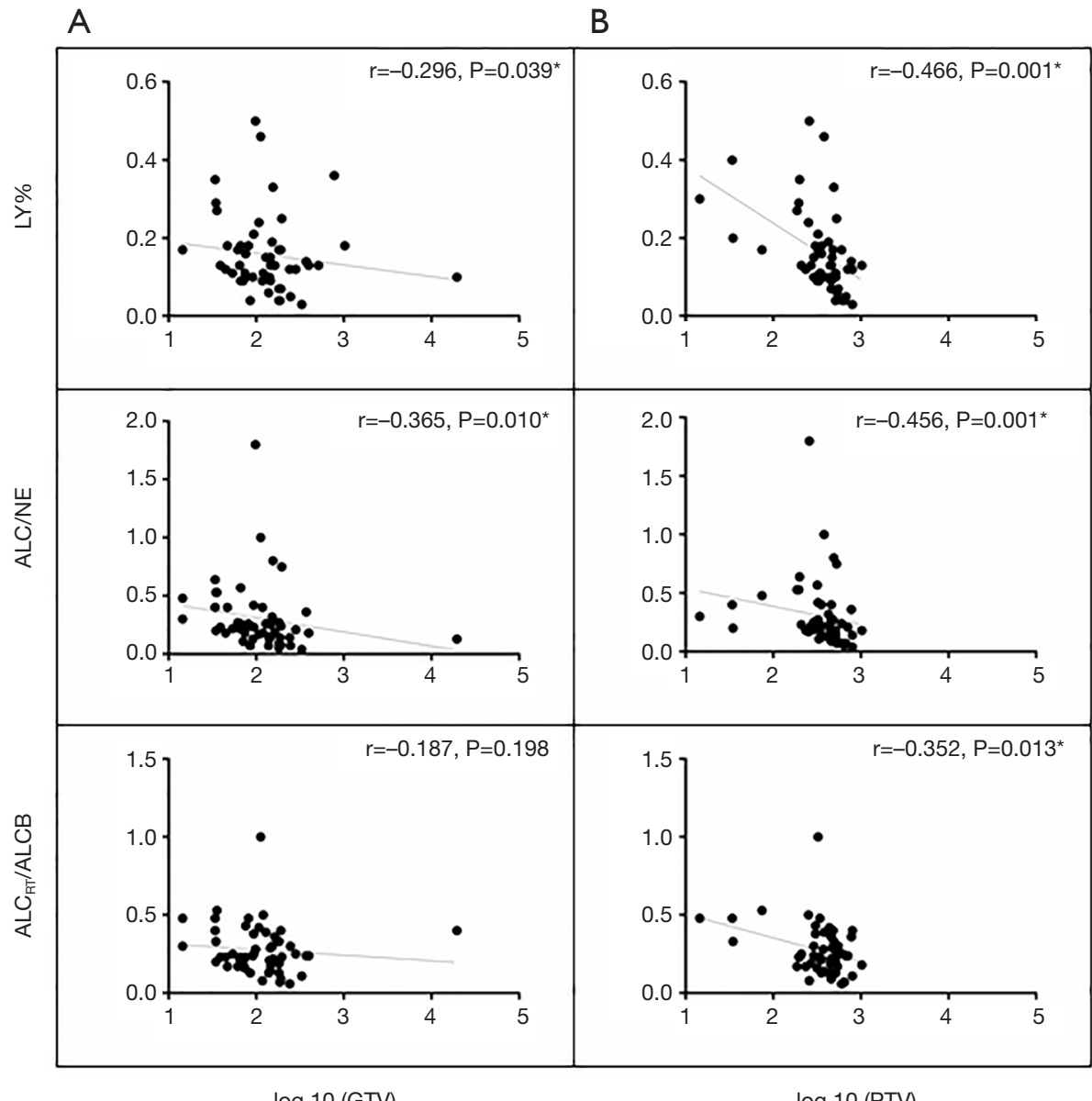

Figure 5 Correlations among the LY\% nadir, ALC/NE nadir during radiotherapy, ALCRT/ALCB with log 10 [GTV (cc)] (A) and log 10 [PTV-g (cc)] (B). Spearman's correlation coefficients and corresponding P values are shown. *, significantly correlated. LY\%, percentage of lymphocytes; NE, neutrophil; ALC, absolute lymphocyte count; GTV, gross tumor volume; r, correlation coefficient; PTV-g, planning target volume without CTV expansion.

PACIFIC trial (12) confirms the synergistic effects of radiotherapy and immunotherapy among patients with LANSCLC. However, radiotherapy is a double-edged sword for immunotherapy. In the PACIFIC trial (12), although severe side effects ( $\geq$ grade 3 ), such as pneumonitis, were not increased by the immunotherapy involved, the total toxicities (all grades) were higher in the combination treatment group. The synergistic interaction between immunotherapy and thoracic irradiation in increasing the risk of pulmonary and cardiac toxicities has also been proven in a series of preclinical models $(29,30)$. As a result, routine toxicities must be continuously investigated in immunotherapy. The original intention of omitting the GTV-to-CTV expansion is to decrease the radiation volume and thus deliver less radiation dose to adjacent normal tissues. This goal has been achieved from the dosimetric perspective (10) and must be similarly applicable when immunotherapy is involved. Radiation exposure also suppresses the immune system, and RIL is especially evident in such conditions. Lymphocytes are important cellular components for immune response, and several studies have revealed that the tumor response to PD-1/ PD-L1 blockade mainly results from recruiting the periphery effective lymphocytes to invade tumors (31). Therefore, we tested the peripheral blood lymphocyte counts during radiotherapy and their associations with target volumes with the aim to elucidate the influences of radiation target volumes to the immune system function. We found that both GTV and PTV were significantly related to lymphopenia during radiotherapy, with larger 
volumes associated with severe lymphopenia. This finding is consistent with a previous report with a larger scale of patients (19). We also found that PTV was more sensitive than GTV to lymphopenia, a conclusion arrived at on the basis of the differences of Spearman's correlation coefficient (r) values between GTV and PTV (Figures 4 and 5). This result arose probably because the variability in PTV was greater than in GTV owing to the change in radius of GTV being cubed when generating PTV. In addition, most tissues between GTV and PTV were normal tissues, where many CLs and normal lymphoid tissues were irradiated during the period. Thus, omitting CTV to reduce PTV could better protect the immune function in radiotherapy, thereby favoring immunotherapy.

In our previous dosimetric study (10), we also tested the simultaneous integrated boost (SIB) technique that enables the intended simultaneous delivery of different doses to different areas of the treatment volume. We found that SIB could improve dose distribution compared with the CTV omission plan for the dose-sparing to the normal tissues while maintaining the adequate doses to the treatment targets. The SIB technique is also feasible in clinical settings for LA-NSCLC (32-36). However, the doses required to eradicate subclinical diseases were mainly derived empirically from preclinical research works $(8,9,37)$ and only few retrospective studies with different dose deliveries (32-36) (the required doses may be lower or higher than 45 $50 \mathrm{~Gy}$ ). Thus, we cannot provide specific recommendations for the SIB technique on the basis of the aforementioned dosimetric study (10), and the present study suggests that further investigation is warranted.

Among the limitations of this study were its retrospective nature, with the associated biases, and its single-center single-arm study with a relatively small sample size. Furthermore, the immunotherapy involved after concurrent chemoradiotherapy is the current standard of care for unresectable LA-NSCLC. However, the treatment schedule of this study was completed before immunotherapy. Therefore, whether the results can be generalized to when immunotherapy is implemented is still questionable, while the beneficial effect of CTV omission for the immune system must be further evaluated in immunotherapy.

In conclusion, we found that CTV omission is feasible for LA-NSCLC treated with concurrent chemoradiotherapy by using the IMRT technique, and the failure inside the subclinical region was not compromised. The radiation volumes were associated with lymphopenia during radiotherapy, with larger volumes related to severe lymphopenia. This finding indicates that CTV omission IMRT should be considered in immunotherapy for further investigation.

\section{Acknowledgments}

Funding: This project was supported by the National Science Foundation of China (No. 81572963 to Zhengfei Zhu).

\section{Footnote}

Reporting Checklist: The authors have completed the STROBE reporting checklist. Available at http://dx.doi. org/10.21037/tlcr-20-523

Data Sharing Statement: Available at http://dx.doi. org/10.21037/tlcr-20-523

Conflicts of Interest: All authors have completed the ICMJE uniform disclosure form (available at http://dx.doi. org/10.21037/tlcr-20-523). The authors have no conflicts of interest to declare.

Ethical Statement: The authors are accountable for all aspects of the work in ensuring that questions related to the accuracy or integrity of any part of the work are appropriately investigated and resolved. The study was conducted in accordance with the Declaration of Helsinki (as revised in 2013). The study was approved by institutional ethics board of Fudan University Shanghai Cancer Center (No. IRB\#090978-2). Patient consent was waived because this study was a retrospective study.

Open Access Statement: This is an Open Access article distributed in accordance with the Creative Commons Attribution-NonCommercial-NoDerivs 4.0 International License (CC BY-NC-ND 4.0), which permits the noncommercial replication and distribution of the article with the strict proviso that no changes or edits are made and the original work is properly cited (including links to both the formal publication through the relevant DOI and the license). See: https://creativecommons.org/licenses/by-nc-nd/4.0/.

\section{References}

1. Herbst RS, Morgensztern D, Boshoff C. The biology and management of non-small cell lung cancer. Nature 
2018;553:446-54.

2. Brown NA, Aisner DL, Oxnard GR. Precision Medicine in Non-Small Cell Lung Cancer: Current Standards in Pathology and Biomarker Interpretation. Am Soc Clin Oncol Educ Book 2018;38:708-15.

3. Song C, Pyo H, Kim J, et al. Superiority of conventional intensity-modulated radiotherapy over helical tomotherapy in locally advanced non-small cell lung cancer. A comparative plan analysis. Strahlenther Onkol 2012;188:901-9.

4. Wang J, Zhou Z, Liang J, et al. Intensity-Modulated Radiation Therapy May Improve Local-Regional Tumor Control for Locally Advanced Non-Small Cell Lung Cancer Compared With Three-Dimensional Conformal Radiation Therapy. Oncologist 2016;21:1530-7.

5. Hudson A, Chan C, Woolf D, et al. Is heterogeneity in stage 3 non-small cell lung cancer obscuring the potential benefits of dose-escalated concurrent chemo-radiotherapy in clinical trials? Lung Cancer 2018;118:139-47.

6. Ma L, Men Y, Feng L, et al. A current review of doseescalated radiotherapy in locally advanced non-small cell lung cancer. Radiol Oncol 2019;53:6-14.

7. Roach MC, Bradley JD, Robinson CG. Optimizing radiation dose and fractionation for the definitive treatment of locally advanced non-small cell lung cancer. J Thorac Dis 2018;10:S2465-73.

8. Withers HR, Peters LJ, Taylor JM. Dose-response relationship for radiation therapy of subclinical disease. Int J Radiat Oncol Biol Phys 1995;31:353-9.

9. Withers HR, Suwinski R. Radiation dose response for subclinical metastases. Semin Radiat Oncol 1998;8:224-8.

10. Xia F, Zhou L, Yang X, et al. Is a clinical target volume (CTV) necessary for locally advanced non-small cell lung cancer treated with intensity-modulated radiotherapy? -a dosimetric evaluation of three different treatment plans. J Thorac Dis 2017;9:5194-202.

11. Antonia SJ, Villegas A, Daniel D, et al. Overall Survival with Durvalumab after Chemoradiotherapy in Stage III NSCLC. N Engl J Med 2018;379:2342-50.

12. Antonia SJ, Villegas A, Daniel D, et al. Durvalumab after Chemoradiotherapy in Stage III Non-Small-Cell Lung Cancer. N Engl J Med 2017;377:1919-29.

13. Spaas M, Lievens Y. Is the Combination of Immunotherapy and Radiotherapy in Non-small Cell Lung Cancer a Feasible and Effective Approach? Front Med (Lausanne) 2019;6:244.

14. Sellins KS, Cohen JJ. Gene induction by gammairradiation leads to DNA fragmentation in lymphocytes. J
Immunol 1987;139:3199-206.

15. Stratton JA, Byfield PE, Byfield JE, et al. A comparison of the acute effects of radiation therapy, including or excluding the thymus, on the lymphocyte subpopulations of cancer patients. J Clin Invest 1975;56:88-97.

16. Ellsworth SG. Field size effects on the risk and severity of treatment-induced lymphopenia in patients undergoing radiation therapy for solid tumors. Adv Radiat Oncol 2018;3:512-9.

17. Campian JL, Ye X, Brock M, et al. Treatment-related lymphopenia in patients with stage III non-small-cell lung cancer. Cancer Invest 2013;31:183-8.

18. McCoy MJ, Lake RA, van der Most RG, et al. Postchemotherapy T-cell recovery is a marker of improved survival in patients with advanced thoracic malignancies. Br J Cancer 2012;107:1107-15.

19. Tang C, Liao Z, Gomez D, et al. Lymphopenia association with gross tumor volume and lung V5 and its effects on non-small cell lung cancer patient outcomes. Int J Radiat Oncol Biol Phys 2014;89:1084-91.

20. Karantanos T, Karanika S, Seth B, et al. The absolute lymphocyte count can predict the overall survival of patients with non-small cell lung cancer on nivolumab: a clinical study. Clin Transl Oncol 2019;21:206-12.

21. Venkatesulu BP, Mallick S, Lin SH, et al. A systematic review of the influence of radiation-induced lymphopenia on survival outcomes in solid tumors. Crit Rev Oncol Hematol 2018;123:42-51.

22. Jouglar E, Isnardi V, Goulon D, et al. Patterns of locoregional failure in locally advanced non-small cell lung cancer treated with definitive conformal radiotherapy: Results from the Gating 2006 trial. Radiother Oncol 2018;126:291-9.

23. Kilburn JM, Lucas JT, Soike MH, et al. Is a Clinical Target Volume (CTV) Necessary in the Treatment of Lung Cancer in the Modern Era Combining 4-D Imaging and Image-guided Radiotherapy (IGRT)? Cureus 2016;8:e466.

24. Liang X, Yu H, Yu R, et al. Efficacy of the smaller target volume for stage III non-small cell lung cancer treated with intensity-modulated radiotherapy. Mol Clin Oncol 2015;3:1172-6.

25. Arriagada R, Bergman B, Dunant A, et al. Cisplatinbased adjuvant chemotherapy in patients with completely resected non-small-cell lung cancer. $\mathrm{N}$ Engl J Med 2004;350:351-60.

26. Winton T, Livingston R, Johnson D, et al. Vinorelbine plus cisplatin vs. observation in resected non-small-cell lung cancer. N Engl J Med 2005;352:2589-97. 
27. Strauss GM, Herndon JE 2nd, Maddaus MA, et al. Adjuvant paclitaxel plus carboplatin compared with observation in stage IB non-small-cell lung cancer: CALGB 9633 with the Cancer and Leukemia Group B, Radiation Therapy Oncology Group, and North Central Cancer Treatment Group Study Groups. J Clin Oncol 2008;26:5043-51.

28. Douillard J. ANITA: phase III adjuvant vinorelbine $(\mathrm{N})$ and cisplatin $(\mathrm{P})$ versus observation (OBS) in completely resected (stage I-III) non-small-cell lung cancer (NSCLC) patients (pts): final results after 70-month median followup. On behalf of the Adjuvant Navelbin. J Clin Oncol 2005;23:abstr 7013.

29. Myers CJ, Lu B. Decreased Survival After Combining Thoracic Irradiation and an Anti-PD-1 Antibody Correlated With Increased T-cell Infiltration Into Cardiac and Lung Tissues. Int J Radiat Oncol Biol Phys 2017;99:1129-36.

30. Lin AJ, Roach M, Bradley J, et al. Combining stereotactic body radiation therapy with immunotherapy: current data and future directions. Transl Lung Cancer Res 2019;8:107-15.

31. Yost KE, Satpathy AT, Wells DK, et al. Clonal replacement of tumor-specific T cells following PD-1 blockade. Nat Med 2019;25:1251-9.

32. Dong Y, Zhang Y, Zhang T, et al. Feasibility and Efficacy of Simultaneous Integrated Boost Intensity-modulated Radiation Therapy based on MRI-CT fusion in Patients with Brain Metastases of Non-small Cell Lung Cancer. J Cancer 2018;9:4477-83.

33. Jeter MD, Gomez D, Nguyen QN, et al. Simultaneous Integrated Boost for Radiation Dose Escalation to the Gross Tumor Volume With Intensity Modulated (Photon) Radiation Therapy or Intensity Modulated Proton Therapy and Concurrent Chemotherapy for Stage II to III Non-Small Cell Lung Cancer: A Phase 1 Study. Int J Radiat Oncol Biol Phys 2018;100:730-7.

34. Ji K, Zhao LJ, Liu WS, et al. Simultaneous integrated boost intensity-modulated radiotherapy for treatment of locally advanced non-small-cell lung cancer: a retrospective clinical study. Br J Radiol 2014;87:20130562.

35. Wang D, Bi N, Zhang T, et al. Comparison of efficacy and safety between simultaneous integrated boost intensitymodulated radiotherapy and conventional intensitymodulated radiotherapy in locally advanced non-smallcell lung cancer: a retrospective study. Radiat Oncol 2019;14:106.

36. Xu Y, Zheng X, Bai X, et al. Simultaneous integrated boost intensity-modulated radiotherapy for locally advanced non-small cell lung cancer in Chinese population: A retrospective study. Oncotarget 2017;8:49084-92.

37. Okunieff P, Morgan D, Niemierko A, Suit HD. Radiation dose-response of human tumors. Int J Radiat Oncol Biol Phys 1995;32:1227-37.
Cite this article as: Zou L, Chu L, Xia F, Zhou L, Yang X, Ni J, Chen J, Zhu Z. Is clinical target volume necessary? - a failure pattern analysis in patients with locally advanced non-small cell lung cancer treated with concurrent chemoradiotherapy using intensity-modulated radiotherapy technique. Transl Lung Cancer Res 2020;9(5):1986-1995. doi: 10.21037/tlcr-20-523 


\section{Supplementary}

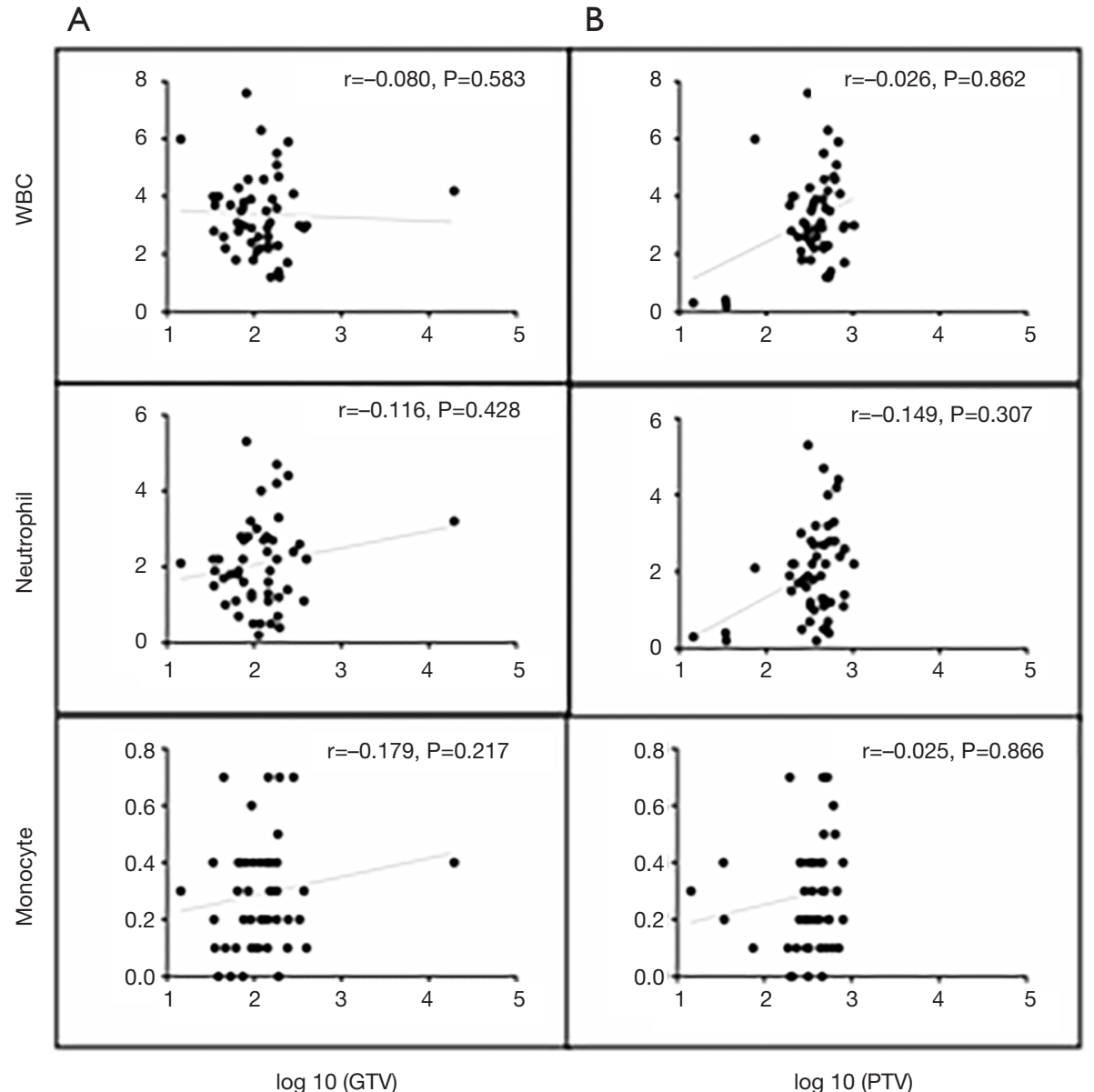

Figure S1 Correlations among nadirs of WBC, neutrophils, and monocytes during radiotherapy and log 10 [GTV (cc)] and log 10 [PTV-g (cc)]. Spearman's correlation coefficients and corresponding P values are shown. WBC, white blood cell; GTV, gross tumor volume; PTV-g, planning target volume without CTV expansion, r, correlation coefficient. 